\title{
A PROSPECTIVE STUDY ON THE SAFETY OF PEDIATRIC CHIROPRACTIC SPINAL MANIPULATIVE THERAPY: RESULTS FROM A PRACTICE-BASED RESEARCH NETWORK
}

\author{
J. Alcantara ${ }^{1,2}$, J. Ohm ${ }^{3,4}$, K. Kunz ${ }^{5}$ \\ ${ }^{1}$ International Chiropractic Pediatric Association, San Jose, ${ }^{2}$ Research, Life Chiropractic College West, \\ Hayward, CA, International Chiropractic Pediatric Association, ${ }^{4}$ Chiropractic, Ohm Chiropractic, \\ ${ }^{5}$ Practice Based Research Network, International Chiropractic Pediatric Association, Media, PA, USA
}

Background: Concerns regarding the safety and effectiveness of complementary and alternative medicine (CAM) therapies have been raised. Of the various practitioner-based CAM therapies, chiropractic remains popular. To address safety concerns, we examined the prevalence and incidence of adverse events in a practice-based research network setting.

Methods: This study was approved by the Institutional Review Board of Life University (Atlanta, GA, USA). In a prospective cohort study, we characterized the chiropractic care of children and examined the prevalence and incidence of adverse events (AEs) associated with pediatric SMT.

Results: Two-hundred sixty-four chiropractors reported on 512 children. An AE prevalence of $0.67 \%$ was calculated with risk estimates of 880,141 and 28 cases per 1 million person-year for the first, second and third AE occurrence. Four hundred forty nine parents reported on similar number of children. A prevalence of $4.45 \%$ calculated with risk estimates of 978 and 172 cases per 1 million person-year for the first and second AE occurrence.

Discussion: Children attend chiropractic care for wellness and to address dysfunctions of the neuromusculoskeletal system and conditions of childhood. Parents indicate a high perceived effectiveness with indicated AEs as minor and self-limiting.

Conclusion: We provided supporting data that AEs associated with pediatric chiropractic SMT are rare. We encourage further research in this field. 